\title{
Unspecified Antepartum Hypertension
}

National Cancer Institute

\section{Source}

National Cancer Institute. Unspecified Antepartum Hypertension. NCI Thesaurus. Code C35222.

High blood pressure in the period of time preceding labor and delivery. 